\title{
Kink-Like Periodic Travelling Waves for Lattice Equations with On-Site and Inter-Site Potentials
}

\author{
Michal Fečkan and Vassilis Rothos
}

Communicated by Michal Fečkan, received July 5, 2005.

\begin{abstract}
The existence of travelling generalized kinks with oscillation tails is studied for a class of $1 \mathrm{D}$ lattice equations with both onsite and intersite potential. The travelling wave equation of the corresponding discrete nonlinear equation is formulated as an advanced-delay differential equation which is reduced by a center manifold method to a 4-dimensional singular ODE with certain symmetries and with a symmetric heteroclinic structure. Bifurcations of solutions from the heteroclinic ones are investigated for the singular perturbation systems of autonomous o.d.eqns in $\mathbb{R}^{4}$. This gives the existence of generalized kink solutions with co-propagating oscillation tails.
\end{abstract}

\section{Contents}

1. Introduction $\quad 357$

2. Formalism in discrete dynamical system 359

3. Center manifold reductions 361

4. Bifurcation Results 363

5. Travelling Waves 365

6. Generalized potentials 366

7. Appendix: Linearization around heteroclinic connection $\quad 367$

$\begin{array}{ll}\text { References } & 370\end{array}$

\section{Introduction}

In recent years there has been a flurry of mathematical research arising from condensed matter physics and physical chemistry, namely the study of localised

1991 Mathematics Subject Classification. Primary 34C23, 34 C25; Secondary 34C37, 35 B99.

Key words and phrases. singular perturbations, bifurcations, travelling waves.

M.F supported by Grant VEGA-SAV 2/4135/25 .

V.R supported by EPSRC Grant No. GR/R02702/01 and LMS Grant Scheme 5. 
modes in anharmonic molecules and molecular crystals. Using classical approximations, these are described by nonlinear lattice equations (differential-difference equations).

Most nonlinear lattice systems are not integrable even if the PDE model in the continuum limit is; see [2], [5] and references therein. Prototype models for such nonlinear lattices take the form of various discrete NLS equations or systems, a particularly important class of solutions of which are so called discrete breathers which are homoclinic in space and oscillatory in time. Other questions involve the existence and propagation of topological defects or kinks which mathematically are heteroclinic connections between a ground and an excited steady state. Nonequilibrium dynamics of many physical systems can be characterized by the creation and motion of topological excitations or defects, so called kinks, which have applications to problems such as dislocation and mass transport in solids, charge-density waves, commensurable-incommensurable phase transitions, Josephson transmission lines etc. Prototype models here are discrete version of sine-Gordon equations, also known as Frenkel-Kontorova (FK) models. There are many outstanding issues for such systems relating to the global existence and dynamics of localised modes for general nonlinearities, away from either continuum or anti-continuum limits.

In this paper, we consider a perturbed Hamiltonian chain of coupled oscillators with an Hamiltonian

$$
\mathcal{H}=\sum_{n \in \mathbb{Z}}\left(\frac{1}{2} \dot{u}_{n}^{2}+\frac{1}{2 \varepsilon^{2}}\left(u_{n+1}-u_{n}\right)^{2}+H_{\alpha}\left(u_{n}\right)+\mu G\left(u_{n+1}-u_{n}\right)\right),
$$

where $\varepsilon>0$ is a discretness parameter and $\mu$ is a small parameter measuring the relation of intersite and offsite potentials, $H_{\alpha}, G \in C^{2}(\mathbb{R})$ and $\alpha \geq 0$ is a parameter. The Hamiltonian $\mathcal{H}$ gives the discrete nonlinear Klein-Gordon eqn:

$$
\begin{gathered}
\ddot{u}_{n}-\frac{1}{\varepsilon^{2}}\left(u_{n+1}-2 u_{n}+u_{n-1}\right)+h_{\alpha}\left(u_{n}\right) \\
+\mu\left\{g\left(u_{n}-u_{n-1}\right)-g\left(u_{n+1}-u_{n}\right)\right\}=0,
\end{gathered}
$$

where $h_{\alpha}(x)=H_{\alpha}^{\prime}(x)$ and $g(x)=G^{\prime}(x)$.

Eqn (1.2) with $\mu=0$ can be considered as a spatial discretization of the p.d.eqn

$$
u_{t t}-u_{\xi \xi}+h_{\alpha}(u)=0 .
$$

The discrete sine-Gordon equation for $h_{0}(u)=\sin u,(\alpha=0)$ and $\mu=0$ in (1.2) of the form

$$
\ddot{u}_{n}=u_{n+1}-2 u_{n}+u_{n-1}-\Gamma^{2} \sin u_{n}
$$

has been numerically investigated by Eilbeck and co-workers $[\mathbf{3}],[\mathbf{9}]:$ As $\Gamma \rightarrow 0$, we get the continuum sine-Gordon equation with the supporting moving kinks of the form

$$
u(x, t)=4 \arctan \left[\exp \left(\Gamma \frac{x-\nu t}{\sqrt{1-\nu^{2}}}\right)\right] .
$$

Thus it was natural [3] to seek numerically solutions of

$$
\nu^{2} U^{\prime \prime}(z)=U(z+1)-2 U(z)+U(z-1)-\Gamma^{2} \sin U(z),
$$

where $U(z)=U(n-\nu t)=u_{n}(t)$, with the boundary conditions $U(z) \rightarrow 0 \bmod 2 \pi$ as $z \rightarrow \pm \infty$. He did not find such solutions. His closest result is that the numerical solution of (1.6) near (1.5) has tails of periodic waves of small amplitude. 
We also consider a Hamiltonian perturbation to (1.4) of the form

$$
\begin{gathered}
\ddot{u}_{n}-\frac{1}{\varepsilon^{2}}\left(u_{n+1}-2 u_{n}+u_{n-1}\right)+\sin u_{n} \\
+\mu\left\{\sin \left(u_{n}-u_{n+1}\right)+\sin \left(u_{n}-u_{n-1}\right)\right\}=0 .
\end{gathered}
$$

Motivated by (1.7), we fix $h \equiv h_{\alpha}$ for $\alpha=0$ and suppose the following conditions:

(A1) $h, g \in C^{1}(\mathbb{R})$ are odd, $h$ is $2 \pi$-periodic, $h(x-\pi)=-h(x)$ and $g$ is globally Lipschitz on $\mathbb{R}$.

(A2) $h(-\pi)=h(\pi)=0, h^{\prime}(-\pi)=h^{\prime}(\pi)=a^{2}>0$ and there is a heteroclinic solution $\Phi$ of $\ddot{x}-h(x)=0$ such that $\Phi(t)=2 \pi-\Phi(-t)$ and $\Phi(t) \rightarrow 2 \pi$ as $t \rightarrow+\infty$.

For (1.7) clearly $h(x)=g(x)=\sin x$ and $\Phi(t)=4 \arctan [\exp t]$. We show bellow in Section 6 that the condition $h(x-\pi)=-h(x)$ is superfluous in (A1), so it can be omitted. But for simplicity, we prove the results under condition (A1).

By assumption (A2), the ODE (1.3) with $\alpha=0$ admits travelling wave solutions

$$
u(x, t)=\Phi\left(\frac{x-\nu t}{\sqrt{1-\nu^{2}}}\right), \quad 0<\nu<1 .
$$

We consider for travelling wave solutions of (1.2) of stationary profile in a moving reference with constant velocity $\nu / \varepsilon$. One can write

$$
u_{n}(t)=V\left(n-\frac{\nu}{\varepsilon} t\right) \equiv V(z), \quad z=n-\frac{\nu}{\varepsilon} t, \quad 0<\nu<1 .
$$

Eqn (1.2) is reduced to the following functional differential equation:

$$
\begin{aligned}
& \nu^{2} V^{\prime \prime}(z)-V(z+1)+2 V(z)-V(z-1)+\varepsilon^{2} h(V(z)) \\
+ & \varepsilon^{2} \mu\{g(V(z)-V(z-1))-g(V(z+1)-V(z))\}=0,
\end{aligned}
$$

where ${ }^{\prime}$ represents differentiation with respect to $z$. This paper provides analytical results about the existence of solutions of eqn (1.8) near $\Phi$ and the relationship between travelling wave solutions of (1.2) and (1.3) for $\varepsilon>0, \mu$ small.

The outline of the paper is as follows: In Section 2, we formulate eqn (1.8) as a dynamical system. In Section 3, we apply center manifold theory to the study of existence of travelling waves with non-small amplitude oscillations on infinite nonlinear lattice (perturbed discrete sine-Gordon). In Sections 4 and 5, we state and prove the main theorems of this paper. In Section 6, we also investigate the existence of travelling wave solutions of eqn (1.7), that are closed to the kink solution when the form of $h_{\alpha}$ is given by

$$
h_{\alpha}(u)=\frac{(1+2 \alpha) \sin u}{(1+\alpha(1-\cos u))^{2}}
$$

and $\alpha \geq 0$. In Appendix, we prove some preliminary results concerning the uniqueness of solution for the linearized o.d.eqn $\ddot{x}+h(x)=0$ along the heteroclinic solution.

\section{Formalism in discrete dynamical system}

In this section, we consider the advanced-delay differential equation (1.8) as a dynamical system. 
We shift $V(z) \longleftrightarrow V(z)-\pi$ and note $h(x-\pi)=-h(x)$ in (1.8) to get the following functional differential equation:

$$
\begin{aligned}
& \nu^{2} V^{\prime \prime}(z)-V(z+1)+2 V(z)-V(z-1)-\varepsilon^{2} h(V(z)) \\
+ & \varepsilon^{2} \mu\{g(V(z)-V(z-1))-g(V(z+1)-V(z))\}=0 .
\end{aligned}
$$

Since computations are the same as in [4], we follow that paper. We introduce a new variable $v \in[-1,1]$ and functions $X(t, v)=x(t+v)$. The notation $U(t)(v)=$ $(x(t), \xi(t), X(t, v))$ indicates our intention to construct $V$ as a map from $\mathbb{R}$ into some function space living on the $v$-interval $[-1,1]$. Eqn $(2.1)$ can be written as follows

$$
U_{t}=L U+\frac{\varepsilon^{2}}{\nu^{2}} M(U)
$$

$$
U(t, v)=(x(t), \xi(t), X(t, v)), \quad v \in[-1,1]
$$

where

$$
\begin{gathered}
L=\left(\begin{array}{ccc}
0 & 1 & 0 \\
-\frac{2}{\nu^{2}} & 0 & \frac{1}{\nu^{2}} \delta^{1}+\frac{1}{\nu^{2}} \delta^{-1} \\
0 & 0 & \partial_{v}
\end{array}\right) \\
M(U)=\left(0, h(x)-\mu\left\{g\left(x-\delta^{-1} X(v)\right)-g\left(\delta^{1} X(v)-x\right)\right\}, 0\right)
\end{gathered}
$$

and $\delta^{ \pm 1}$ be the difference operators, defined by $\delta^{ \pm 1} X(v)=X( \pm 1)$. We introduce the Banach spaces $\mathbb{H}$ and $\mathbb{D}$ for $U(v)=(x, \xi, X(v))$

$$
\begin{gathered}
\mathbb{H}=\mathbb{R}^{2} \times C[-1,1], \\
\mathbb{D}=\left\{U \in \mathbb{R}^{2} \times C^{1}[-1,1] \mid X(0)=x\right\}
\end{gathered}
$$

with the usual maximum norms. Then $L \in \mathcal{L}(\mathbb{D}, \mathbb{H})$ and $M \in C^{1}(\mathbb{D}, \mathbb{D})$. We consider $(2.2)$ on $\mathbb{D}$. The spectrum $\sigma(L)$ is given by the explicit solution of the problem $(2.2)$ with $\varepsilon=0$ :

$$
U(t, v)=(x, \xi, X(v)) e^{\lambda t}
$$

where $\lambda$ is given by the roots of characteristic equation:

$$
N(\lambda)=\lambda^{2}+\frac{2}{\nu^{2}}(1-\cosh \lambda)=0
$$

There are infinitely many isolated eigenvalues $\lambda \in \mathbb{C}$. We are interested in those eigenvalues $\lambda$, which define the center manifold of the problem (2.2) at $\Re \lambda=0$. Clearly $\sigma(L)$ is invariant under $\lambda \rightarrow \bar{\lambda}$ and $\lambda \rightarrow-\lambda$. The central part $\sigma_{0}(L)=$ $\sigma(L) \cap \imath \mathbb{R}$ is determined by the equation

$$
q^{2}+\frac{2}{\nu^{2}}(\cos q-1)=0, \quad q \in \mathbb{R} .
$$

The resolvent equation

$$
(\lambda I-L) U=F, \quad \lambda \in \mathbb{C}, U \in \mathbb{D}
$$

has to be solvabled for any given $F \in \mathbb{H}$. When $\lambda$ is not in the spectrum of the operator $L$, the inhomogeneous problem can be solved. The eigenvalues $\lambda$, defined by the roots of the characteristic equation, appear as poled in the solution 
of the resolvent equation. The center manifold reductions follow from the Laurent expansion of the solution of resolvent equation near the eigenvalues $\lambda$ with $\Re \lambda=0$.

The basic properties of $\sigma(L)$ are given in Lemma 1 of $[\mathbf{6}]$ and we refer the reader to that paper for more details. In this paper, we assume that $\nu_{1}<\nu<1$ where $\nu=\nu_{1}$ is the first value from the left of 1 for which the equations

$$
\lambda^{2}+\frac{2}{\nu^{2}}(\cos \lambda-1)=0, \quad \lambda-\frac{1}{\nu^{2}} \sin \lambda=0
$$

have a common nonzero solution $\lambda \neq 0$. Then equation $N(\imath q)=0$ has the double root 0 and simple roots $\pm q$. Hence we have $\sigma_{0}(L)=\{0, \pm \imath q\}$.

\section{Center manifold reductions}

The linear operator on the 4th-dimensional central subspace $\mathbb{H}_{c}$ has the form

$$
L_{c}=L / \mathbb{H}_{c}=\left(\begin{array}{cccc}
0 & 1 & 0 & 0 \\
0 & 0 & 0 & 0 \\
0 & 0 & 0 & q \\
0 & 0 & -q & 0
\end{array}\right)
$$

in the basis $\left(\xi_{1}, \xi_{2}, \xi_{3}, \xi_{4}\right)$ defined by

$$
\begin{array}{cl}
\xi_{1}=(1,0,1), & \xi_{2}=(0,1, v) \\
\xi_{3}=(1,0, \cos q v), \quad \xi_{4}=(0, q, \sin q v)
\end{array}
$$

and which satisfies $L \xi_{1}=0, L \xi_{2}=\xi_{1}, L \xi_{3}=-q \xi_{4}, L \xi_{4}=q \xi_{3}$.

The projection $P_{c}: \mathbb{H} \rightarrow \mathbb{H}_{c}$ is given by

$$
P_{c}(U)=P_{1}(U) \xi_{1}+P_{2}(U) \xi_{2}+P_{3}(U) \xi_{3}+P_{4}(U) \xi_{4}
$$

where

$$
\begin{gathered}
P_{1}(U)=\frac{\nu^{2}}{\nu^{2}-1} x-\frac{1}{\nu^{2}-1} \int_{0}^{1}(1-s)[X(s)+X(-s)] d s, \\
P_{2}(U)=\frac{\nu^{2}}{\nu^{2}-1} \xi+\frac{1}{\nu^{2}-1} \int_{0}^{1}[X(-s)-X(s)] d s \\
P_{3}(U)=\left(\nu^{2} q x-\int_{0}^{1} \sin q(1-s)[X(s)+X(-s)] d s\right) /\left(q \nu^{2}-\sin q\right), \\
P_{4}(U)=\left(\nu^{2} \xi+\int_{0}^{1} \cos q(1-s)[X(-s)-X(s)] d s\right) /\left(q \nu^{2}-\sin q\right) .
\end{gathered}
$$

These projections are derived as the residues of the inverse $(\lambda I-L)^{-1}$ at $\lambda=0, \pm \imath q$, respectively, of the resolvent operator [7].

Condition (A1) implies that $M$ is globally Lipschitz. So we can apply the procedure of a center manifold method [7] to get for $\varepsilon, \mu$ small the reduced equation 
of (2.2) over $\mathbb{H}_{c}$ given by

$$
\begin{aligned}
\dot{u}_{c} & =L_{c} u_{c}+\frac{\varepsilon^{2}}{\nu^{2}} P_{c} M\left(u_{c}+\varepsilon^{2} \Psi_{\varepsilon, \mu}\left(u_{c}\right)\right) \\
& =L_{c} u_{c}+\frac{\varepsilon^{2}}{\nu^{2}} P_{c}\left(M\left(u_{c}\right)\right)+O\left(\varepsilon^{4}\right),
\end{aligned}
$$

where $u_{c}=u_{1} \xi_{1}+u_{2} \xi_{2}+u_{3} \xi_{3}+u_{4} \xi_{4}$ and $\Psi_{\varepsilon, \mu}$ is the graph map of the center manifold. Then (3.1) has the form

$$
\begin{gathered}
\dot{u}_{1}=u_{2}, \quad \dot{u}_{2}=\frac{\varepsilon^{2}}{\nu^{2}-1} \hat{h}\left(u_{1}, u_{2}, u_{3}, u_{4}, \varepsilon^{2}, \mu\right) \\
\dot{u}_{3}=q u_{4}, \quad \dot{u}_{4}=-q u_{3}+\frac{\varepsilon^{2}}{q \nu^{2}-\sin q} \hat{h}\left(u_{1}, u_{2}, u_{3}, u_{4}, \varepsilon^{2}, \mu\right),
\end{gathered}
$$

for a $C^{1}$-function $\hat{h}$. Let us consider

$$
\begin{gathered}
x(t)=x_{1}(t)=u_{1}(t / \varepsilon), \quad x_{2}(t)=u_{2}(t / \varepsilon) / \varepsilon, \\
y(t)=y_{1}(t)=u_{3}(t / \varepsilon), \quad y_{2}(t)=u_{4}(t / \varepsilon) .
\end{gathered}
$$

Then (3.1) has the form

$$
\begin{gathered}
\dot{x}_{1}=x_{2}, \quad \dot{x}_{2}=\frac{1}{\nu^{2}-1} \hat{h}\left(x_{1}, \varepsilon x_{2}, y_{1}, y_{2}, \varepsilon^{2}, \mu\right) \\
\dot{y}_{1}=\frac{q}{\varepsilon} y_{2}, \quad \dot{y}_{2}=-\frac{q}{\varepsilon} y_{1}+\frac{\varepsilon}{q \nu^{2}-\sin q} \hat{h}\left(x_{1}, \varepsilon x_{2}, y_{1}, y_{2}, \varepsilon^{2}, \mu\right),
\end{gathered}
$$

which gives

$$
\begin{gathered}
\ddot{x}=\frac{1}{1-\nu^{2}} f(x, \varepsilon \dot{x}, y, \varepsilon \dot{y} / q, \varepsilon, \mu), \\
\varepsilon^{2} \ddot{y}+q^{2} y=\frac{\varepsilon^{2} q}{\sin q-\nu^{2} q} f(x, \varepsilon \dot{x}, y, \varepsilon \dot{y} / q, \varepsilon, \mu),
\end{gathered}
$$

where $f\left(x_{1}, x_{2}, y_{1}, y_{2}, \varepsilon, \mu\right)=-h\left(x_{1}+y_{1}\right)+O\left(\varepsilon^{2}+|\mu|\right)$. For $\varepsilon=\mu=0$ and $y=0$, the limit equation of (3.2) has the form

$$
\left(1-\nu^{2}\right) \ddot{x}-h(x)=0
$$

which is precisely the travelling wave equation of the PDE (1.3) with $\alpha=0$ shifted by $u \longleftrightarrow u-\pi$. Equation (3.3) has a heteroclinic solution $x(t)=\phi\left(t / \sqrt{1-\nu^{2}}\right.$ ) for $\phi(t)=\Phi(t)-\pi$.

We consider the symmetry $S(U)=(x,-\xi, X(-v))$ on $\mathbb{H}$. Then $(2.2)$ is reversible with respect to $S$, i.e. $S \circ L=-L \circ S, M \circ S=-S \circ M$. Moreover, we have $P_{c} \circ S=S \circ P_{c}$ and $S \xi_{1}=\xi_{1}, S \xi_{2}=-\xi_{2}, S \xi_{3}=\xi_{3}, S \xi_{4}=-\xi_{4}$. Hence

$$
S_{c}=S / \mathbb{H}_{c}=\left(\begin{array}{cccc}
1 & 0 & 0 & 0 \\
0 & -1 & 0 & 0 \\
0 & 0 & 1 & 0 \\
0 & 0 & 0 & -1
\end{array}\right) \text {. }
$$

Since $S_{c}$ is unitary, the map $\Psi_{\varepsilon, \mu}$ can be chosen $[6]$ in such a way that $S \circ \Psi_{\varepsilon, \mu}=$ $\Psi_{\varepsilon, \mu} \circ S_{c}$. This implies

$$
L_{c} S_{c} u_{c}+\frac{\varepsilon^{2}}{\nu^{2}} P_{c} M\left(S_{c} u_{c}+\varepsilon^{2} \Psi_{\varepsilon, \mu}\left(S_{c} u_{c}\right)\right)=-S_{c}\left(L_{c} u_{c}+\frac{\varepsilon^{2}}{\nu^{2}} P_{c} M\left(u_{c}+\varepsilon^{2} \Psi_{\varepsilon, \mu}\left(u_{c}\right)\right)\right) .
$$


Hence (3.1) is reversible with respect to $S_{c}$. Moreover, $S_{c}$ has in the coordinates $\left(x_{1}, x_{2}, y_{1}, y_{2}\right)$ on $\mathbb{H}_{c}$ the form $S_{c}\left(x_{1}, x_{2}, y_{1}, y_{2}\right)=\left(x_{1},-x_{2}, y_{1},-y_{2}\right)$. Consequently we get for $(3.2)$ :

(B1) $f\left(x_{1},-x_{2}, y_{1},-y_{2}, \varepsilon, \mu\right)=f\left(x_{1}, x_{2}, y_{1}, y_{2}, \varepsilon, \mu\right)$.

Furthermore, we consider the symmetry $\tilde{S}(U)=-U$ on $\mathbb{H}$. Then $(2.2)$ is symmetric with respect to $\tilde{S}$, i.e. $\tilde{S} \circ L=L \circ \tilde{S}, M \circ \tilde{S}=\tilde{S} \circ M$. Moreover, we have $P_{c} \circ \tilde{S}=\tilde{S} \circ P_{c}$. Consequently we get for (3.2):

(B2) $f\left(-x_{1},-x_{2},-y_{1},-y_{2}, \varepsilon, \mu\right)=-f\left(x_{1}, x_{2}, y_{1}, y_{2}, \varepsilon, \mu\right)$.

Finally, we consider the shift $\bar{S}(U)=U+2 \pi \xi_{1}$ on $\mathbb{H}$. Then $L=L \circ \bar{S}$, $M \circ \bar{S}=M, P_{c} \circ \bar{S}=\bar{S} \circ P_{c}$. Hence we get $\bar{S} \mathbb{H}_{c}=\mathbb{H}_{c}$ and in the coordinates $\left(x_{1},, x_{2}, y_{1}, y_{2}\right)$ on $\mathbb{H}_{c}$ we have $\bar{S}_{c}\left(x_{1}, x_{2}, y_{1}, y_{2}\right)=\left(x_{1}+2 \pi, x_{2}, y_{1}, y_{2}\right)$ for $\bar{S}_{c}=\bar{S} / \mathbb{H}_{c}$. Consequently we get for (3.2):

(B3) $f\left(x_{1}+2 \pi, x_{2}, y_{1}, y_{2}, \varepsilon, \mu\right)=f\left(x_{1}, x_{2}, y_{1}, y_{2}, \varepsilon, \mu\right)$.

Summarizing we see that the reduced o.d.eqn (3.2) satisfies properties (B1-B3).

\section{Bifurcation Results}

Motivated by properties (B1-3), we study in this section singularly perturbed systems of the form

$$
\begin{gathered}
\ddot{x}+h(x)=f_{1}(x, \dot{x}, y, \varepsilon \dot{y}, \varepsilon), \\
\varepsilon^{2} \ddot{y}+y=\varepsilon^{2} g_{1}(x, \dot{x}, y, \varepsilon \dot{y}, \varepsilon),
\end{gathered}
$$

where $\varepsilon>0$ is a small parameter and we assume the following assumptions

(C1) $f_{1}, g_{1} \in C^{1}, f_{1}\left(x_{1}, x_{2}, 0,0,0\right)=0$.

(C2) $f_{1}\left(x_{1},-x_{2}, y_{1},-y_{2}, \varepsilon\right)=f_{1}\left(x_{1}, x_{2}, y_{1}, y_{2}, \varepsilon\right)=-f_{1}\left(-x_{1}, x_{2},-y_{1}, y_{2}, \varepsilon\right)=$ $f_{1}\left(x_{1}+2 \pi, x_{2}, y_{1}, y_{2}, \varepsilon\right)$ and $g_{1}\left(x_{1},-x_{2}, y_{1},-y_{2}, \varepsilon\right)=g_{1}\left(x_{1}, x_{2}, y_{1}, y_{2}, \varepsilon\right)=$ $-g_{1}\left(-x_{1}, x_{2},-y_{1}, y_{2}, \varepsilon\right)=g_{1}\left(x_{1}+2 \pi, x_{2}, y_{1}, y_{2}, \varepsilon\right)$.

We note that (A3) implies the next property

(C3) $h(-\pi)=h(\pi)=0, h^{\prime}(-\pi)=h^{\prime}(\pi)=-a^{2}<0$ and $\phi(t)=\Phi(t)-\pi$ is a heteroclinic solution of $\ddot{x}+h(x)=0$ such that $\Phi(-t)=-\Phi(t)$ and $\Phi(t) \rightarrow \pi$ as $t \rightarrow+\infty$.

We studied in [4] a similar problem when equation $\ddot{x}+h(x)=0$ had a homoclinic solution.

First we are looking for periodic solutions of (4.1) near a heteroclinic loop $(\phi(t), 0) \cup(\phi(-t), 0)$. For this reason, we make the change of variables

$$
x(t)=\phi(t)+\varepsilon^{1 / 4} u(t), \quad y(t)=\sqrt{\varepsilon} v(t),
$$

and we get

$$
\begin{gathered}
\varepsilon^{2} \ddot{v}+v=\varepsilon^{3 / 2} g_{1}\left(\phi+\varepsilon^{1 / 4} u, \dot{\phi}+\varepsilon^{1 / 4} \dot{u}, \sqrt{\varepsilon} v, \varepsilon^{3 / 2} \dot{v}, \varepsilon\right) \\
\ddot{u}+h^{\prime}(\phi) u=-\frac{1}{\varepsilon^{1 / 4}}\left\{h\left(\phi+\varepsilon^{1 / 4} u\right)-h(\phi)-h^{\prime}(\phi) \varepsilon^{1 / 4} u\right\} \\
+\frac{1}{\varepsilon^{1 / 4}} f_{1}\left(\phi+\varepsilon^{1 / 4} u, \dot{\phi}+\varepsilon^{1 / 4} \dot{u}, \sqrt{\varepsilon} v, \varepsilon^{3 / 2} \dot{v}, \varepsilon\right) .
\end{gathered}
$$


We are looking for solutions of (4.1) satisfying $x(0)=\dot{x}(T)=0, y(0)=\dot{y}(T)=0$. This gives

$$
\begin{gathered}
u(0)=0, \quad \dot{u}(T)=-\dot{\phi}(T) / \varepsilon^{1 / 4} \\
v(0)=0, \quad \dot{v}(T)=0 .
\end{gathered}
$$

The next result deals with this problem.

Theorem 4.1. For any $k_{0} \in \mathbb{N}$ there is an $\varepsilon_{0}>0$ such that for any $0<\varepsilon<\varepsilon_{0}$ and $T=\varepsilon\left(k\left[\varepsilon^{-3 / 2}\right] \pi+\tau\right)$ with $k \in \mathbb{N}, k \leq k_{0}, \tau \in[-\pi / 3, \pi / 3]$, system (4.1) has a $4 T$-periodic solution $(x(t), y(t))$ near $(\phi(t), 0),-T \leq t \leq T$ such that $x, y$ are odd functions and $x(t+2 T)=-x(t), y(t+2 T)=-y(t)$. Here $\left[\varepsilon^{-3 / 2}\right]$ is the integer part of $\varepsilon^{-3 / 2}$.

Proof. First of all, by using Lemmas 7.1-7.2 from Appendix and the approach as in the first part of the proof of Theorem 3.1 in [4], we see that for any $k_{0} \in \mathbb{N}$ there is an $\varepsilon_{0}>0$ such that for any $0<\varepsilon<\varepsilon_{0}$ and $T=\varepsilon\left(k\left[\varepsilon^{-3 / 2}\right] \pi+\tau\right)$ with $k \in \mathbb{N}$, $k \leq k_{0}, \tau \in[-\pi / 3, \pi / 3]$, problem (4.2)-(4.3) has a solution on the interval $[0, T]$. This gives a solution of (4.1) near $(\phi(t), 0), 0 \leq t \leq T$ satisfying $x(0)=\dot{x}(T)=0$ and $y(0)=\dot{y}(T)=0$. We extend these functions as follows

$$
x(t)=\left\{\begin{array}{l}
x(t) \text { for } t \in[0, T] \\
x(2 T-t) \text { for } t \in[T, 2 T] \\
-x(t-2 T) \text { for } t \in[2 T, 3 T] \\
-x(4 T-t) \text { for } t \in[3 T, 4 T],
\end{array}\right.
$$

and

$$
y(t)=\left\{\begin{array}{l}
y(t) \text { for } t \in[0, T] \\
y(2 T-t) \text { for } t \in[T, 2 T] \\
-y(t-2 T) \text { for } t \in[2 T, 3 T] \\
-y(4 T-t) \text { for } t \in[3 T, 4 T]
\end{array}\right.
$$

We easily check that these are the desired $4 T$-periodic solutions stated in Theorem 4.1 .

Now we are looking for a solution near a heteroclinic solution $(\phi(t), 0)$. For this reason we consider the conditions $x(0)=0, x(T)=\pi$ and $y(0)=y(T)=0$. The above change of variables gives

$$
\begin{gathered}
u(0)=0, \quad u(T)=(\pi-\phi(T)) / \varepsilon^{1 / 4} \\
v(0)=v(T)=0 .
\end{gathered}
$$

So we consider the problem (4.2) subject to (4.4). The next result deals with this problem.

TheOREM 4.2. For any $k_{0} \in \mathbb{N}$ there is an $\varepsilon_{0}>0$ such that for any $0<\varepsilon<\varepsilon_{0}$ and $T=\varepsilon\left(k\left[\varepsilon^{-3 / 2}\right] \pi+\tau\right)$ with $k \in \mathbb{N}, k \leq k_{0}, \tau \in[\pi / 6, \pi / 2]$, system (4.1) has a solution $(x(t), y(t))$ on $\mathbb{R}$ near $(\phi(t), 0),-T \leq t \leq T$ such that $x, y$ are odd functions and $x(t+2 T)=x(t)+2 \pi, y(t+2 T)=y(t)$.

Proof. Again, by using Lemmas 7.3-7.4 from Appendix and the approach as in the first part of the proof of Theorem 3.1 in [4], we see that for any $k_{0} \in \mathbb{N}$ there is an $\varepsilon_{0}>0$ such that for any $0<\varepsilon<\varepsilon_{0}$ and $T=\varepsilon\left(k\left[\varepsilon^{-3 / 2}\right] \pi+\tau\right)$ with $k \in \mathbb{N}$, $k \leq k_{0}, \tau \in[\pi / 6, \pi / 2]$, problem (4.2) with (4.4) has a solution on the interval $[0, T]$. 
This gives a solution of (4.1) near $(\phi(t), 0), 0 \leq t \leq T$ satisfying $x(0)=0, x(T)=\pi$ and $y(0)=y(T)=0$. We extend these functions as follows

$$
x(t)=\left\{\begin{array}{l}
x(t) \text { for } t \in[0, T] \\
2 \pi-x(2 T-t) \text { for } t \in[T, 2 T]
\end{array}\right.
$$

and

$$
y(t)=\left\{\begin{array}{l}
y(t) \text { for } t \in[0, T] \\
-y(2 T-t) \text { for } t \in[T, 2 T] .
\end{array}\right.
$$

We easily check that these are the desired solutions stated in Theorem 4.2.

REMARK 4.3. We note that the derived $4 T$-periodic solutions $x_{T, \varepsilon}$ and $y_{T, \varepsilon}$ in Theorem 4.1 of equation (4.1) are near to $(\phi(t), 0)$ in the sense that $x_{T, \varepsilon}(t)-\phi(t)=$ $O\left(\varepsilon^{1 / 4}\right), \dot{x}_{T, \varepsilon}(t)-\dot{\phi}(t)=O\left(\varepsilon^{1 / 4}\right), y_{T, \varepsilon}(t)=O(\sqrt{\varepsilon}), \varepsilon \dot{y}_{T, \varepsilon}(t)=O(\sqrt{\varepsilon})$ uniformly for $-T \leq t \leq T$ and $T$ satisfying the assumption of Theorem 4.1 for a fixed $k_{0}$. These estimates are consistent with the form of (4.1). Similarly for Theorem 4.2.

\section{Travelling Waves}

By applying Theorems 4.1 and 4.2 to (3.2) we get the following result:

TheOREM 5.1. For any $k_{0} \in \mathbb{N}$ there is an $\varepsilon_{0}>0$ such that for any $0<\varepsilon<\varepsilon_{0}$, $|\mu| \leq \varepsilon_{0} \varepsilon^{1 / 4}$ and $T=\varepsilon\left(k\left[\varepsilon^{-3 / 2}\right] \pi+\tau\right)$ with $k \in \mathbb{N}, k \leq k_{0}, \tau \in[\pi / 3, \pi / 6]$, system (3.2) has a 4T-periodic solution $\left(x_{T, \varepsilon, 1}(t), y_{T, \varepsilon, 1}(t)\right)$ near $(\phi(t), 0),-T \leq$ $t \leq T$ such that $x_{T, \varepsilon, 1}, y_{T, \varepsilon, 1}$ are odd functions and $x_{T, \varepsilon, 1}(t+2 T)=-x_{T, \varepsilon, 1}(t)$, $y_{T, \varepsilon, 1}(t+2 T)=-y_{T, \varepsilon, 1}(t)$. Moreover, under these assumptions, system (3.2) has a solution $\left(x_{T, \varepsilon, 2}(t), y_{T, \varepsilon, 2}(t)\right)$ on $\mathbb{R}$ near $(\phi(t), 0),-T \leq t \leq T$ such that $x_{T, \varepsilon, 2}, y_{T, \varepsilon, 2}$ are odd functions and $x_{T, \varepsilon, 2}(t+2 T)=x_{T, \varepsilon, 2}(t)+2 \pi, y_{T, \varepsilon, 2}(t+2 T)=y_{T, \varepsilon, 2}(t)$.

The solutions of Theorem 5.1 have the forms

$$
u_{c, i}^{T, \varepsilon}(t)=x_{T, \varepsilon, i}(\varepsilon t) \xi_{1}+\varepsilon \dot{x}_{T, \varepsilon, i}(\varepsilon t) \xi_{2}+y_{T, \varepsilon, i}(\varepsilon t) \xi_{3}+\varepsilon\left(\dot{y}_{T, \varepsilon, i}(\varepsilon t) / q\right) \xi_{4}, \quad i=1,2
$$

in (3.1). Furthermore, we have $U(t, \cdot)=u_{c}(t)+\varepsilon^{2} \Psi_{\varepsilon, \mu}\left(u_{c}(t)\right)=u_{c}(t)+O\left(\varepsilon^{2}\right)$ for (2.2) on the center manifold considered in (3.1). We also note that the $x(t)$ coordinate of $U(t, v)$ in (2.2) satisfies (2.1). Consequently, if $x^{T, \varepsilon, i}(\varepsilon t), i=1,2$ are the $x$-coordinates of $u_{c, i}^{T, \varepsilon}(t)+\varepsilon^{2} \Psi_{\varepsilon, \mu}\left(u_{c, i}^{T, \varepsilon}(t)\right)$, then the travelling wave solutions of (1.2) corresponding to $x_{T, \varepsilon, i}(t), y_{T, \varepsilon, i}(t)$ have the forms

$$
\begin{gathered}
u_{n}^{T, \varepsilon, i}(t)=x^{T, \varepsilon, i}\left(\varepsilon\left(n-\frac{\nu}{\varepsilon} t\right)\right)=x^{T, \varepsilon, i}(\varepsilon n-\nu t)= \\
x_{T, \varepsilon, i}(\varepsilon n-\nu t)+y_{T, \varepsilon, i}(\varepsilon n-\nu t)+O\left(\varepsilon^{2}\right) .
\end{gathered}
$$

Clearly $u_{n}^{T, \varepsilon, 1}(t)$ is $T / \nu$-periodic in $t$ with the velocity $\nu$ while $u_{n}^{T, \varepsilon, 2}(t)$ is $T / \nu$ periodically shifted (librational) in $t$ with the velocity $\nu$. Then Remark 4.3 gives

$$
u_{n}^{T, \varepsilon, i}(t)=\phi\left(\frac{\varepsilon n-\nu t}{\sqrt{1-\nu^{2}}}\right)+O\left(\varepsilon^{1 / 4}\right)
$$

uniformly for $-T \leq \varepsilon n-\nu t \leq T$ and $T$ satisfying the assumptions of Theorem 5.1 for a fixed $k_{0}$. We shall call travelling wave solution $u_{n}^{T, \varepsilon, 1}(t)+\pi, n \in \mathbb{Z}$ of (1.2) as rotational while $u_{n}^{T, \varepsilon, 2}(t)+\pi, n \in \mathbb{Z}$ as librational.

Finally, we note that we get (1.2) with $\mu=0$ from (1.3) by putting

$$
\begin{gathered}
u_{n}(t)=u(\varepsilon n, t), \\
u_{x x}(\varepsilon n, t) \sim \frac{u(\varepsilon(n+1), t)-2 u(\varepsilon n, t)+u(\varepsilon(n-1), t)}{\varepsilon^{2}} .
\end{gathered}
$$


Summarizing we get the main analytical result of this paper.

THEOREM 5.2. If $h, g$ satisfy the assumptions $(A 1-2)$ then travelling wave solution $u(x, t)=\Phi\left(\frac{x-\nu t}{\sqrt{1-\nu^{2}}}\right)$ for $0<\nu_{1}<\nu<1$ of (1.3) with $\alpha=0$ can be approximated by the both rotational and librational travelling wave solutions of (1.2) with $\alpha=0$ with very large periods and with the velocity $\nu$ for $\mu=o\left(\varepsilon^{1 / 4}\right)$ small.

We note that travelling wave solution (5.1) of (1.8) derived in this paper have tails of periodic waves of small amplitude caused by the $y$-components in (5.1). This result is consistent with the numerical result of J.C. Eilbeck for (1.6) mentioned in Introduction.

For $0<\nu<\nu_{1}$, we could still use the above method. We know from [6] that there is a decreasing sequence $\left\{\nu_{i}\right\}_{i=1}^{\infty} \subset(0,1)$ with $\nu_{i} \rightarrow 0$ as $i \rightarrow \infty$ and for any $\nu_{i+1}<\nu<\nu_{i}$ the linear operator $L$ has the double non semi-simple eigenvalue at 0 , and $2 i+1$ pairs of simple imaginary eigenvalues. So after the center manifold reduction, we should get a system like (3.2) and we could generalize the bifurcation results of Section 4 for such systems. We do not carry out those computations in this paper.

\section{Generalized potentials}

We note in this section that our method can be used for broader class of functions $h$ than above. For instance, let us consider P.D.E.

$$
u_{t t}-u_{x x}+h_{\alpha}(u)=0
$$

for

$$
h_{\alpha}(u)=\frac{(1+2 \alpha) \sin u}{(1+\alpha(1-\cos u))^{2}}
$$

and $\alpha \geq 0$. We note that $h_{\alpha}(u-\pi) \neq-h_{\alpha}(u)$ for $\alpha>0$, so condition (A1) does not hold. But still like in (1.7), we take its spatial discretization with a Hamiltonian perturbation of the form

$$
\begin{gathered}
\ddot{u}_{n}-\frac{1}{\varepsilon^{2}}\left(u_{n+1}-2 u_{n}+u_{n-1}\right)+h_{\alpha}\left(u_{n}\right) \\
+\mu\left\{\sin \left(u_{n}-u_{n+1}\right)+\sin \left(u_{n}-u_{n-1}\right)\right\}=0 .
\end{gathered}
$$

Now we make a change of variables $u \longleftrightarrow u-\pi$ and $u_{n} \longleftrightarrow u_{n}-\pi$ in (6.1) and (6.2), respectively, to get

$$
u_{t t}-u_{x x}+g_{\alpha}(u)=0
$$

and

$$
\begin{gathered}
\ddot{u}_{n}-\frac{1}{\varepsilon^{2}}\left(u_{n+1}-2 u_{n}+u_{n-1}\right)+g_{\alpha}\left(u_{n}\right) \\
+\mu\left\{\sin \left(u_{n}-u_{n+1}\right)+\sin \left(u_{n}-u_{n-1}\right)\right\}=0
\end{gathered}
$$

respectively, for

$$
g_{\alpha}(u)=-\frac{(1+2 \alpha) \sin u}{(1+\alpha(1+\cos u))^{2}}
$$


Clearly $g_{\alpha} \in C^{1}(\mathbb{R})$ is odd and $2 \pi$-periodic. Hence we can carry out the center manifold reduction of Section 3 for (6.2) to get a system like (4.1) with the nonsingular unperturbed part

$$
\left(1-\nu^{2}\right) \ddot{x}-g_{\alpha}(x)=0 .
$$

We have $g_{\alpha}(-\pi)=g_{\alpha}(\pi)=0$ and $g_{\alpha}^{\prime}(-\pi)=g^{\prime}{ }_{\alpha}(\pi)=1+2 \alpha>0$. Hence $(-\pi, 0)$ and $(\pi, 0)$ are hyperbolic equilibria of

$$
\dot{x}=y, \quad \dot{y}=g_{\alpha}(x) .
$$

It is not difficult to observe that the upper odd heteroclinic solution $\phi_{\alpha}$ of (6.6) connecting $(-\pi, 0)$ and $(\pi, 0)$ is determined by the equation

$$
\begin{aligned}
& \dot{\phi}_{\alpha}=2 \sqrt{1+2 \alpha} \frac{\cos \left(\phi_{\alpha} / 2\right)}{\sqrt{1+2 \alpha \cos ^{2}\left(\phi_{\alpha} / 2\right)}}, \\
& \phi_{\alpha}(0)=0,
\end{aligned}
$$

which is equivalent to the implicit equation

$$
\sqrt{2 \alpha} \arcsin \frac{\sqrt{2 \alpha} \sin \left(\phi_{\alpha}(t) / 2\right)}{\sqrt{1+2 \alpha}}+\operatorname{arctanh} \frac{\sin \left(\phi_{\alpha}(t) / 2\right)}{\sqrt{1+2 \alpha \cos ^{2}\left(\phi_{\alpha}(t) / 2\right)}}=\sqrt{1+2 \alpha} .
$$

Summarizing, we can apply the results of the above sections to (6.2) uniformly for $\alpha \geq 0$ from bounded intervals. Moreover, we see that our method can be used when instead of the condition $h(x-\pi)=-h(x)$ we consider $h(-x-\pi)=-h(x-\pi)$. But if $h$ is odd and $2 \pi$-periodic then $h(-x-\pi)=h(-x+\pi)=-h(x-\pi)$. Hence the condition $h(x-\pi)=-h(x)$ is superfluous in (A1), so it can be omitted.

\section{Appendix: Linearization around heteroclinic connection}

We take the linearization of the equation

$$
\ddot{x}+h(x)=0
$$

along $\phi(t)=\Phi(t)-\pi$ and consider the variational equation

$$
\ddot{u}+h^{\prime}(\phi(t)) u=z(t), \quad 0 \leq t \leq T .
$$

We note that $\dot{\phi}(t)$ is even while $\ddot{\phi}(t)$ is odd and $\phi(t)$ satisfies (7.1). Since $h^{\prime}(-\pi)=$ $h^{\prime}(\pi)=-a^{2}<0, a>0$, we have $\dot{\phi}(t), \ddot{\phi}(t) \sim \mathrm{e}^{-a t}$ as $t \rightarrow+\infty$, i.e. it holds that

$$
\dot{\phi}(t) / \mathrm{e}^{-a t} \rightarrow k_{1} \neq 0 \quad \text { and } \quad \ddot{\phi}(t) / \mathrm{e}^{-a t} \rightarrow k_{2} \neq 0 \quad \text { as } \quad t \rightarrow+\infty .
$$

The homogeneous eqn (7.2) with $z=0$ has solutions $w_{i}(t), i=1,2$ such that:

. $w_{1}$ is even, $w_{1}(0)=1, \dot{w}_{1}(0)=0, w_{1}(t), \dot{w}_{1}(t) \sim \mathrm{e}^{-a t}$ as $t \rightarrow+\infty$,

. $w_{2}$ is odd, $w_{2}(0)=0, \dot{w}_{2}(0)=1, w_{2}(t), \dot{w}_{2}(t) \sim \mathrm{e}^{a t}$ as $t \rightarrow+\infty$.

First we consider (7.2) with the boundary value conditions

$$
u(0)=0, \quad \dot{u}(T)=b .
$$

The general solution of (7.2) has the form

$$
\begin{gathered}
u(t)=c_{1} w_{1}(t)+c_{2} w_{2}(t)+z_{1}(t), \\
z_{1}(t)=\int_{0}^{t}\left[w_{2}(t) w_{1}(s)-w_{1}(t) w_{2}(s)\right] z(s) d s .
\end{gathered}
$$


The condition (7.3) gives $c_{1}=0$ and

$$
c_{2}=-\frac{\dot{z}_{1}(T)}{\dot{w}_{2}(T)}+\frac{b}{\dot{w}_{2}(T)} \text {. }
$$

Hence, we get

$$
\begin{gathered}
u(t)=L_{T}^{1}(z, b) \equiv b \frac{w_{2}(t)}{\dot{w}_{2}(T)}-\int_{t}^{T} w_{2}(t) w_{1}(s) z(s) d s \\
+\frac{w_{2}(t)}{\dot{w}_{2}(T)} \int_{0}^{T} \dot{w}_{1}(T) w_{2}(s) z(s) d s-\int_{0}^{t} w_{1}(t) w_{2}(s) z(s) d s .
\end{gathered}
$$

Then

$$
\begin{gathered}
\dot{u}(t)=b \frac{\dot{w}_{2}(t)}{\dot{w}_{2}(T)}-\int_{t}^{T} \dot{w}_{2}(t) w_{1}(s) z(s) d s \\
+\frac{\dot{w}_{2}(t)}{\dot{w}_{2}(T)} \int_{0}^{T} \dot{w}_{1}(T) w_{2}(s) z(s) d s-\int_{0}^{t} \dot{w}_{1}(t) w_{2}(s) z(s) d s .
\end{gathered}
$$

By using the above asymptotic properties of $w_{1}$ and $w_{2}$, there is a constant $C_{1}>0$ such that for any $t, s \in[0, T]$ and $T>0$ large, we get

$$
\begin{gathered}
\left|w_{2}(t) / \dot{w}_{2}(T)\right| \leq C_{1} \mathrm{e}^{a(t-T)}, \quad\left|\dot{w}_{2}(t) / \dot{w}_{2}(T)\right| \leq C_{1} \mathrm{e}^{a(t-T)}, \\
\left|w_{2}(t) w_{1}(s)\right| \leq C_{1} \mathrm{e}^{a(t-s)}, \quad\left|\dot{w}_{2}(t) w_{1}(s)\right| \leq C_{1} \mathrm{e}^{a(t-s)} \\
\left|w_{2}(t) \dot{w}_{1}(T) w_{2}(s) / \dot{w}_{2}(T)\right| \leq C_{1} \mathrm{e}^{a(t+s-2 T)} \\
\left|\dot{w}_{2}(t) \dot{w}_{1}(T) w_{2}(s) / \dot{w}_{2}(T)\right| \leq C_{1} \mathrm{e}^{a(t+s-2 T)} \\
\left|w_{1}(t) w_{2}(s)\right| \leq C_{1} \mathrm{e}^{a(s-t)}, \quad\left|\dot{w}_{1}(t) w_{2}(s)\right| \leq C_{1} \mathrm{e}^{a(s-t)}
\end{gathered}
$$

These estimates imply the existence of a constant $c>0$ such that

$$
\|u\|+\|\dot{u}\| \leq c(|b|+\|z\|),
$$

where $\|x\|=\max _{[0, T]}|x(t)|$. Summarizing, we get the next result.

Lemma 7.1. Problem (7.2)-(7.3) has a unique solution $u=L_{T}^{1}(z, b)$ satisfying (7.4).

Now, we consider the problem

$$
\begin{gathered}
\varepsilon^{2} \ddot{v}+v=\varepsilon z(t), \quad 0 \leq t \leq T, \\
v(0)=\dot{v}(T)=0 .
\end{gathered}
$$

We can immediately see that the solution of eqn (7.5) is given by

$$
v(t)=L_{\varepsilon, T}^{1}(z) \equiv-\frac{\sin (t / \varepsilon)}{\cos (T / \varepsilon)} \int_{0}^{T} \cos \frac{T-s}{\varepsilon} z(s) d s+\int_{0}^{t} \sin \frac{t-s}{\varepsilon} z(s) d s .
$$

If $T$ satisfies

$$
\left|\frac{T}{\varepsilon}-k \pi\right| \leq \pi / 3, \quad k \in \mathbb{N}
$$


then $1 \geq|\cos (T / \varepsilon)| \geq 1 / 2$, and we obtain the estimate

$$
\|v\|+\|\varepsilon \dot{v}\| \leq 6 T\|z\|
$$

Summarizing, we get the next result.

LEMMA 7.2. If condition (7.6) holds then problem (7.5) has a unique solution $v=L_{\varepsilon, T}^{1}(z)$ satisfying $(7.7)$.

Next, we consider (7.2) with the boundary conditions

$$
u(0)=0, \quad u(T)=b .
$$

By substituting (7.8) into the above general solution of (7.2), we get $c_{1}=0$ and $c_{2}=\frac{b}{w_{2}(T)}-\frac{z_{1}(T)}{w_{2}(T)}$. Hence, we get

$$
\begin{gathered}
u(t)=L_{T}^{2}(z, b) \equiv b \frac{w_{2}(t)}{w_{2}(T)}-\int_{t}^{T} w_{2}(t) w_{1}(s) z(s) d s \\
+\frac{w_{2}(t)}{w_{2}(T)} \int_{0}^{T} w_{1}(T) w_{2}(s) z(s) d s-\int_{0}^{t} w_{1}(t) w_{2}(s) z(s) d s .
\end{gathered}
$$

Then

$$
\begin{gathered}
\dot{u}(t)=b \frac{\dot{w}_{2}(t)}{w_{2}(T)}-\int_{t}^{T} \dot{w}_{2}(t) w_{1}(s) z(s) d s \\
+\frac{\dot{w}_{2}(t)}{w_{2}(T)} \int_{0}^{T} w_{1}(T) w_{2}(s) z(s) d s-\int_{0}^{t} \dot{w}_{1}(t) w_{2}(s) z(s) d s .
\end{gathered}
$$

Again by using the above asymptotic properties of $w_{1}$ and $w_{2}$, there is a constant $C_{2}>0$ such that for any $t, s \in[0, T]$ and $T>0$ large, we get

$$
\begin{gathered}
\left|w_{2}(t) / w_{2}(T)\right| \leq C_{2} \mathrm{e}^{a(t-T)}, \quad\left|\dot{w}_{2}(t) / w_{2}(T)\right| \leq C_{2} \mathrm{e}^{a(t-T)}, \\
\left|w_{2}(t) w_{1}(s)\right| \leq C_{2} \mathrm{e}^{a(t-s)}, \quad\left|\dot{w}_{2}(t) w_{1}(s)\right| \leq C_{2} \mathrm{e}^{a(t-s)} \\
\left|w_{2}(t) w_{1}(T) w_{2}(s) / w_{2}(T)\right| \leq C_{2} \mathrm{e}^{a(t+s-2 T)} \\
\left|\dot{w}_{2}(t) w_{1}(T) w_{2}(s) / w_{2}(T)\right| \leq C_{2} \mathrm{e}^{a(t+s-2 T)} \\
\left|w_{1}(t) w_{2}(s)\right| \leq C_{2} \mathrm{e}^{a(s-t)}, \quad\left|\dot{w}_{1}(t) w_{2}(s)\right| \leq C_{2} \mathrm{e}^{a(s-t)}
\end{gathered}
$$

These estimates imply the existence of a constant $\bar{c}>0$ such that

$$
\|u\|+\|\dot{u}\| \leq \bar{c}(|b|+\| z||) .
$$

Summarizing, we get the next result.

LEMMA 7.3. Problem (7.2)-(7.8) has a unique solution $u=L_{T}^{2}(z, b)$ satisfying (7.9).

Finally, we consider the problem

$$
\begin{gathered}
\varepsilon^{2} \ddot{v}+v=\varepsilon z(t), \quad 0 \leq t \leq T, \\
v(0)=v(T)=0 .
\end{gathered}
$$


We can immediately see that the solution of eqn (7.10) is given by

$$
v(t)=L_{\varepsilon, T}^{2}(z) \equiv-\frac{\sin (t / \varepsilon)}{\sin (T / \varepsilon)} \int_{0}^{T} \sin \frac{T-s}{\varepsilon} z(s) d s+\int_{0}^{t} \sin \frac{t-s}{\varepsilon} z(s) d s .
$$

If $T$ satisfies

$$
\pi / 2 \geq\left|\frac{T}{\varepsilon}-k \pi\right| \geq \pi / 6, \quad k \in \mathbb{N}
$$

then $1 \geq|\sin (T / \varepsilon)| \geq 1 / 2$, and we obtain the estimate

$$
\|v\|+\|\varepsilon \dot{v}\| \leq 6 T\|z\| \text {. }
$$

Summarizing, we get the next result.

LEMMA 7.4. If condition (7.11) holds then problem (7.10) has a unique solution $v=L_{\varepsilon, T}^{2}(z)$ satisfying $(7.12)$.

\section{References}

1. A.A. Aigner, A.R. Champneys and V.M. Rothos, A new barrier to the existence of moving kinks in Frenkel-Kontorova lattices, Physica D 186 (2003), 148-170.

2. S. Aubry and R.S. MacKay, Proof of existence of breathers for time-reversible or Hamiltonian networks of weakly coupled oscillators, Nonlinearity 6 (1994), 1623-1643.

3. J. C. Eilbeck and R. Flesch, Calculation of families of solitary waves on discrete lattices, Physics Letters A 149 (1990), 200-202.

4. M. Fečkan and V. Rothos, Bifurcations of periodics from homoclinics in singular o.d.e.: Applications to discretizations of travelling waves of p.d.e., Comm. Pure Appl. Anal. 1 (2002), 475-483.

5. S. Flach and C.R. Willis, Discrete breathers, Phys. Rep. 295 (1998), 181-264.

6. G. Iooss, Travelling waves in the Fermi-Pasta Ulam lattice, Nonlinearity 13 (3) (2000), 849866.

7. G. Iooss and K. Kirchgässner, Traveling waves in a chain of coupled nonlinear oscillators, Comm. Math. Phys. 211 (2) (2000), 439-464.

8. A.V. Savin, Y. Zolotaryuk and J.C. Eilbeck, Moving kinks and nanopterons in the nonlinear Klein-Gordon lattices, Physica D 138 (2000), 267-281.

9. A. Scott, Nonlinear Sciences: Emergence and Dynamics of Coherent Structures, Oxford Texts Appl. Eng. Math. Nonl. Sciences, vol. 8, Oxford Univ. Press, 2nd edition, 2003.

Department of Mathematical Analysis and Numerical Mathematics, Comenius UniVersity, Mlynská dolina, 84248 Bratislava, Slovakia and Mathematical Institute, Slovak Academy of Sciences, Štefánikova 49, 81473 Bratislava, Slovakia

E-mail address: Michal.Feckan@fmph.uniba.sk

School of Mathematical Sciences, Queen Mary, University of London, London E1 $4 \mathrm{NS}, \mathrm{UK}$

E-mail address: V.M.Rothos@qmul.ac.uk 